\title{
Consensus Control for a Network of Multiple Elastic Joints Robots with Algebraic Riccati Equation (Are)
}

\author{
A.S.M.Isira, N.S.Hamdan, W.C.Yan, Hairi.M.H
}

\begin{abstract}
This paper deals with the leader-follower consensus problem of Lipschitz nonlinear systems under fixed directed communication networks specifically for multiple Elastic Joints Robots. A State-feedback consensus controller is proposed for the system, based on state and output measurements of neighbouring agents, respectively. The controller gain is obtained with Algebraic Riccati Equation (ARE) protocol. An example is included to demonstrate the effectiveness of the state consensus controller. It can be shown that both controllers are stable and able to reach consensus for the system despite the influence of the nonlinear Lipschitz element from the robot.

Keywords: Lipschitz; Nonlinear; Controller; Stability
\end{abstract}

\section{INTRODUCTION}

$\mathrm{I}_{\mathrm{n}}$ n recent years, distributed cooperative control of multi-agents system has attracted a lot of attention from researchers of different fields, such as synchronization [1], formation control [2], multiple mobile robot systems [3], and distributed sensor networks [4]. In the context of multi-agents system, each member of the system is known as an agent or subsystem with the same characteristics of dynamics in a group known as a multi-agents system. In numerous applications, the subsystems or agents are required to achieve the same task or to reach an agreement upon certain quantities of interest, which is referred to as consensus or agreement. One significant advance in consensus control is to use tools in graph theory, especially the use of Laplacian matrices, to characterize the network connection.

According to the characteristics of the multi-agents system, a multi-agents network can be classified to three categories; first-order (single integrator), second-order (double integrator), and high-order. The dynamics of each agent in the first-order system is relatively simple. However, to achieve a more accurate and effective control action the system should describe in complex system as second-order or high-order.

General high-order linear system, whose consensus realization criteria is more difficult to be determined since each agent does not have specific any structure features as

Revised Manuscript Received on October 22, 2019.

* Correspondence Author

A.S.M.Isira*, Fakulti Kejuruteraan Elektronik dan Kejuruteraan Komputer, Email: sadhiqin@utem.edu.my

N.S.Hamdan, Fakulti Kejuruteraan Elektronik dan Kejuruteraan Komputer. Universiti Teknikal Malaysia Melaka, Melaka, Malaysia. Email: nursyahirah21296@gmail.com

W.C. Yan, School of Aerospace Engineering, Beijing Institute of Technology Beijing, China. Email: chunyan.wang@bit.edu.cn

M.H. Hairi, Fakulti Kejuruteraan Elektik, Universiti Teknikal Malaysia Melaka, Melaka, Malaysia. Email: hendra@utem.edu.my simple as the first-order system. Note that in the literature, many results reported were mainly about the consensus control for different kind of multiple dynamical systems, for example for single-integrator and second-order multi-agent dynamical systems in [5], [6] and for high order multi-agent dynamical system in [7], [8].

The network links between the agents is the main feature for the consensus structure analysis and controller design. In graph theory, Laplacian matrix is often used to indicate the network connections in multi-agents system. A theoretical framework for consensus problems of multi-agent systems with fixed and switching topologies was presented in [9],[10] The results were extended in [11], in which a unified approach for the consensus of multi-agent systems with general linear node dynamics was introduced.

Early results on consensus problems were mostly limited to linear dynamics and less on nonlinear systems. The difficulty of consensus control for nonlinear systems owes to certain restrictions the nonlinearity imposes when using the information of the individual systems. Consensus control for second-order Lipschitz nonlinear multi-agent systems was addressed in [12]- [14]. The consensus problem of high-order multiagent systems with nonlinear dynamics was studied in [15]- [17]. For the practical application, two real physical systems that are closely resembled the Lipschitz nonlinear system such as Chua's circuit [18] and the flexible robot [19]. Several control strategies have been employed for both systems since both systems were published.

In consensus control, certain publications such as [20], [21] used flexible mobile robot to demonstrate their ideas in consensus control. Based on the same paper and Spong [19], this paper will concentrate on an example of flexible robot formations to demonstrate our ideas to solve consensus problem. Two contributions can be made from this paper:

1) A state feedback consensus controller is applied on Spong's model [19] that resembles a nonlinear system with Lipschitz nonlinearity. This type of controller has never been applied on Spong's model before.

2) The outcome of the simulation example proves that the controller is stable, and the system achieves consensus.

The arrangement of the paper is as follows: Section 1 is the introduction of the paper. Section 2 provides the fundamental background of consensus control. Section 3 explains the Spong's model for Elastic Joint Robot together with the controller applied on the system. 
Section 4 shows simulation results for state feedback consensus controller application for Elastic Joint Robot network system that includes Lipschitz nonlinearity element. Finally, Section 5 concludes the paper.

\section{PROBLEM STATEMENT}

Consider $\mathrm{N}+1$ nonlinear subsystem with identical dynamics, described as

$$
\begin{aligned}
& x_{i}=A_{i}+\emptyset\left(x_{i}\right)+B u_{i} \\
& y_{i}=C x_{i}
\end{aligned}
$$

where for $i=0, \ldots, N, x_{i} \in R^{n}$ is the state vector of the subsystem, $u_{i} \in R^{p}$ is the input of the $i$ th subsystem, and $y_{i} \in R^{q}$ is the measured output vector, $A \in R^{n \times n}$, $B \in R^{n \times p}$, and $C \in R^{q \times n}$ are appropriate matrices, and $\phi: R^{n} \rightarrow R^{n}$ is a Lipschitz nonlinear function with the Lipschitz constant $Y$, i.e. for any two constant vectors $a, b \in R^{n}$, we have

$$
\|\varnothing(a)-\emptyset(b)\| \leq y\|a-b\|
$$

Without loss of generality, a subsystem indexed by 0 is assigned as the leader with $u_{0}=0$ and the subsystems indexed by $i=1, \ldots, N$ are referred to as followers.

The connections between the subsystems are specified by a directed graph $G$ which consists of a set of vertices denoted by $\nu$ and a set of edges denoted by $\mathcal{E}$. A vertex represents a subsystem and each edge represents a connection. The adjacency matrix $Q$ associated with the graph $G$ is defined with elements $q_{i j}=1$ if there is a connection between subsystem $j$ to $i$, and $q_{i j}=0$ otherwise. The Laplacian matrix $\mathcal{L}=\left\{l_{i j}\right\}$ is commonly defined as

$$
\begin{aligned}
& l_{i j}=-q_{i j i} \text { if } j \neq i \\
& l_{i i}=\sum_{j=1 j}^{N}{ }_{1 j} q_{i j}
\end{aligned}
$$

A few assumptions are needed.

Assumption 1: Triple matrices $(A, B, C)$ are controllable and observable.

Assumption 2: $\quad$ A leader subsystem is fixed.

Assumption 3: The communication network $G$ of the multi-agent systems contains a directed spanning tree with the leader as the root.

Since the leader has no neighbors, $\mathcal{L}$ can be partitioned as

$$
L=\left[\begin{array}{cc}
0 & 0_{1 x N} \\
l_{1} & \bar{L}
\end{array}\right]
$$

where $\overline{\mathcal{L}} \in R^{N \times N}$ and $h_{1}=\left[l_{10}, \ldots, l_{\text {No }}\right]^{T} \in R^{N \times I}$.

Then we need Lemma 1

Lemma 1 [19]: If the Assumption 3 holds, then all the eigenvalues of $\bar{L}$ have positive real parts.
The control objective is to design the leader-follower consensus controller using measured output information of the neighboring agents such that the states of the follower subsystems in a group asymptotically track the state of the leader subsystem.

\section{A. Elastic Joint Robot Model}

A model used is the elastic joint robot, as shown in Figure 1. It is a single-link manipulator with revolute joints actuated by a DC motor. The elasticity of the joint can be modelled as a linear tensional spring, and the elastic coupling of the motor shaft to the link introduces an additional degree of freedom [19]. The states of the system are motor position, the link position and velocity.

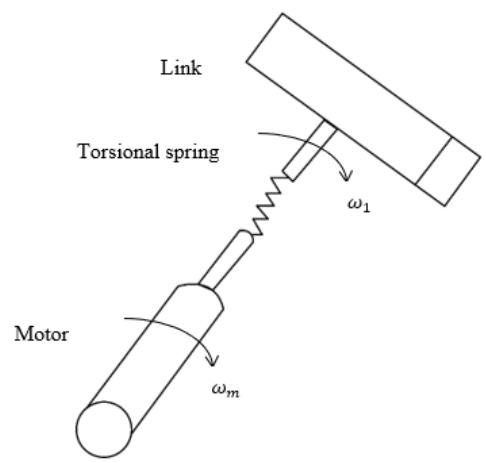

Figure 1: Elastic Joint Robot

The corresponding state-space model is

$$
\begin{gathered}
\theta_{m}=\omega_{m} \\
\omega_{m}=\frac{k}{J_{m}}\left(\theta_{1}-\theta_{m}\right)-\frac{B}{J_{m}} \omega_{m}+\frac{K_{\tau}}{J_{m}} u \\
\theta_{1}=\omega_{1} \\
\omega_{1}=\frac{k}{J_{1}}\left(\theta-\theta_{m}\right)-\frac{m g h}{J_{1}} \sin \theta_{1}
\end{gathered}
$$

with $I_{m}$ being the inertia of the motor, $h_{1}$ the inertia of the link, $\theta_{m}$ the angular rotation of the motor, $\theta_{1}$ the angular position of the link, $\omega_{m}$ the angular velocity of the motor, and $\omega_{1}$ the angular velocity of the link.

These describes the system as nonlinear, and the system dynamics are

$$
\dot{x}=A_{x}+\emptyset(x)+B u ; y=C x
$$

with $x=\left[\begin{array}{llll}\theta_{m} & \omega_{m} & \theta_{1} & \omega_{1}\end{array}\right]^{T}$

$$
A=\left[\begin{array}{cccc}
0 & 1 & 0 & 0 \\
-48.6 & -1.25 & 48.6 & 0 \\
0 & 0 & 0 & 1 \\
19.5 & 0 & -19.5 & 0
\end{array}\right] ; \quad B=\left[\begin{array}{c}
0 \\
21.6 \\
0 \\
0
\end{array}\right]
$$

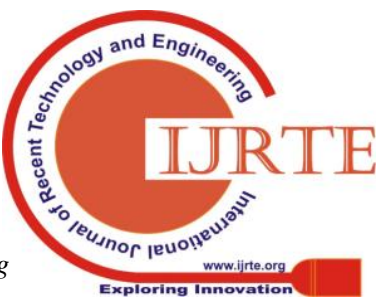




$$
\phi(x)=\left[\begin{array}{c}
0 \\
0 \\
0 \\
\gamma \sin x_{9}
\end{array}\right] ; \quad C=\left[\begin{array}{llll}
1 & 0 & 0 & 0 \\
0 & 1 & 0 & 0
\end{array}\right]
$$

Spong [19] presented a nonlinear I/O linearizing control law for this system. The control law guarantees closed-loop stability and tracking ability for any desired trajectory of the robotic link. However, Spong's work was in single system. It is interesting to see if a number of flexible mobile robot's parameters controlled with consensus controller in a distributed manner. Therefore, the next example will demonstrate our idea of distributed control on flexible mobile robot with the application of state-feedback consensus designed later.

\section{SIMULATION AND RESULTS}

The nonlinear system dynamics (8) can be transferred to a multi-agents system dynamics in the form of nonlinear system (1) with the same matrices $A_{x} B, C$ and $\phi\left(x_{i}\right)$ for all $i=1_{s} \ldots, N$ where $N$ represents the number of agents. Let $x_{i 1}(t)$ be the angular rotation of the motor, $x_{i 2}(t)$ be the angular velocity of the motor, $x_{i 3}(t)$ be the angular rotation of the link of the $i^{\text {th }}$ manipulator, and $x_{i 4}$ be the angular velocity of the link of the $i^{\text {th }}$ manipulator. $\gamma$ was determined to be 0.333 , with $\kappa_{1}=1$ and $\kappa_{2}=3$. It can be verified that matrices $(A, B, C)$ are controllable and observable.

In this example, state-feedback consensus controller is applied. The connections between agents is shown in Figure 2 and the Laplacian matrix obtained is (14). Note that the controller applied is from [22] using the relative state information which takes the structure

$$
u_{i}=K \sum_{j=0}^{N} l_{i j} x_{j}=K \sum_{j=1}^{N} l_{i j}\left(x_{j}-x_{0}\right)
$$

where $x_{0}$ is the leader, $K \in R^{p \times n}$ is a constant control gain matrix to be designed later and $l_{i 0}=-\sum_{j=1}^{Q} l_{i j}$.

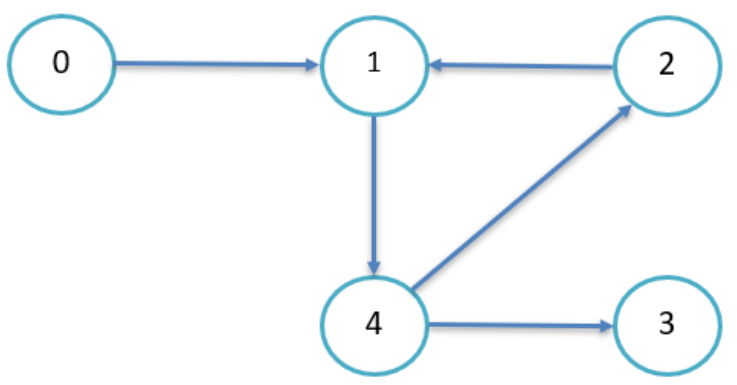

Figure 2: Connections Between Agents

The leader-follower consensus problem is said to be solved if

$$
x_{i}(t) \rightarrow x_{0}(t), \quad \forall i=1_{x \ldots s} N \text { as } t \rightarrow \infty
$$

The solution of $P$ is obtained with Algebraic Riccati Equation (ARE) procedure by obtaining the Hamiltonian matrix from MATLAB/Simulink. This matrix needs to have no eigenvalues on the imaginary axis [24]. Hence, we can obtain eigenvalues of the Hamiltonian matrix $H_{1}$ to be $9.8574,-9.8574,2.8793+5.4484 i, 2.8793-5.4484 i$,
$-2.8793+5.4484 i,-2.8793-5.4484 i, 1.0130$, and -1.0130 . We then obtained the controller gain matrix (from $K=B^{T} P$ ) as

$$
K=\left[\begin{array}{llll}
0.7044 & 0.1533 & -0.4935 & 0.0886
\end{array}\right]
$$

where

$$
P=\left[\begin{array}{cccc}
4.3414 & 0.0326 & -3.1209 & 0.5353 \\
0.0326 & 0.0071 & -0.0228 & 0.0041 \\
-3.1209 & -0.0228 & 3.8188 & -0.2690 \\
0.5353 & 0.0041 & -0.2690 & 0.2898
\end{array}\right]
$$

Similarly, simulations were done with Simulink/MATLAB. The plots obtained for $\$ \backslash$ gamma $=0.333 \$$ are shown in Figure 3 and Figure 4. When $Y$ was increased to 0.8 , it is observed that the subsystem still reached consensus after small oscillations. The signal was smooth when $\gamma=0.333$. However, as we increased $\gamma$ further to $\gamma>1.2$, the system became unstable.

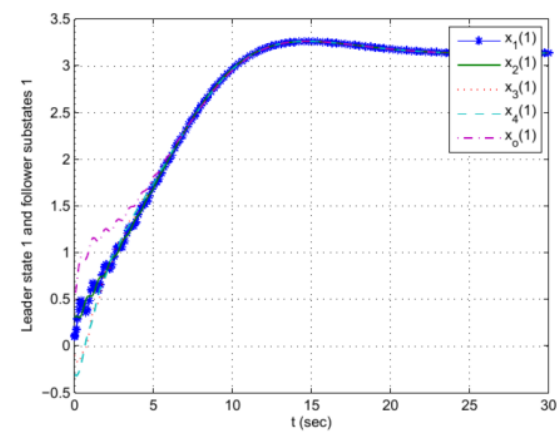

(a)

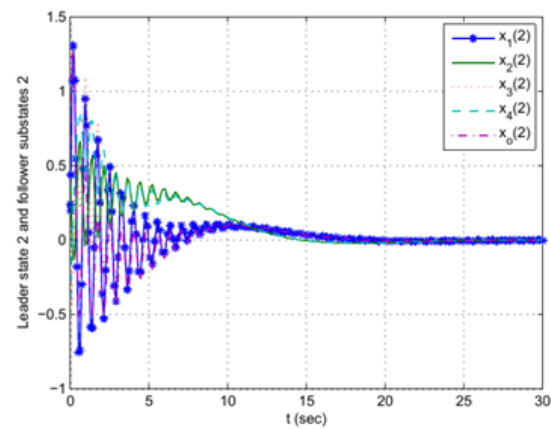

(b)

Figure 3: (a) Plot of leader substate 1 and follower substates 1 consensus state feedback controller, under the influence of Lipschitz nonlinearity at $\gamma=0.333$. (b) Plot of Leader substate 2 and follower substates 2 consensus state feedback controller, under the influence of Lipschitz nonlinearity at $\$ \backslash$ gamma $=0.333 \$$.

Based on the simulation plots, it is clear that, the consensus is successfully reached with the application of controller (10). The lipschitz nonlinearity range that is covered for this simulation is relatively small from $0.1 \leq \gamma \leq 1.2$ where it is evident that the system is stable. This shows that the elastic joint robot system is able to withstand the influence of the nonlinearity element from the motor within a quantifiable range. ARE protocol [24] is used to obtain the values of matrix $P$ for controller matrix $K$. 


\section{CONCLUSION}

In this paper has provided an example of consensus control application for a flexible joint robot with state-feedback consensus controller. The solution for matrix $\mathrm{P}$ for both controller gains were obtained using ARE procedure. The elastic joint robot system with the controller designed showed that consensus has been successfully reached from the simulations shown. This proved that the controller is stable and able to withstand Lipschitz nonlinearity element in the system. For future research, the state-feedback controller can be compared with observer-based consensus controller for further observation and verification. The inclusion of observer in controller design is highly suitable when a system's states are mostly unmeasurable and need to be estimated.

\section{ACKNOWLEDGMENT}

The first author would like to thank Universiti Teknikal Malaysia Melaka (UTeM) for the sponsorship. Grant: PJP/2018/FKEKK(7C)/S01620.

\section{REFERENCES}

1. E. Garcia, Y. Cao, and D. W. Casbeer, "Periodic event-triggered synchronization of linear multi-agent systems with communication delays," IEEE Transactions on Automatic Control, vol. 62, no. 1, pp. 366-371, Jan 2017

2. Z. Liu, Y. Wang, and X. Yang, "Information consensus-based formation control of unmanned surface vehicles," in 2017 36th Chinese Control Conference (CCC), July 2017, pp. 8074-8077.

3. S. Seghour and M. Tadjine, "Consensus-based approach and reactive fuzzy navigation for multiple no-holonomic mobile robots," in 2017 6th International Conference on Systems and Control (ICSC), May 2017, pp. 492-497.

4. J. Qin, W. Fu, H. Gao, and W. X. Zheng, "Distributed k -means algorithm and fuzzy c -means algorithm for sensor networks based on multiagent consensus theory," IEEE Transactions on Cybernetics, vol. 47, no. 3, pp.772-783, March 2017.

5. H. Du, J. Zhou, D. Wu, and G. Wen, "Consensus for second-order nonlinear leader-following multi-agent systems via event-triggered control," in 2017 International Workshop on Complex Systems and Networks (IWCSN), Dec 2017, pp. 301-305.

6. C. Ren, Z. Shi, and T. Du, "Distributed observer-based leader-following consensus control for second-order stochastic multi-agent systems," IEEE Access, vol. 6, pp. 20 077-20 084, 2018.

7. S. Su and Z. Lin, "Distributed consensus control of multi-agent systems with higher order agent dynamics and dynamically changing directed interaction topologies," IEEE Transactions on Automatic Control, vol. 61, no. 2, pp. 515-519, Feb 2016.

8. C. L. P. Chen, G. Wen, Y. Liu, and Z. Liu, "Observer-based adaptive backstepping consensus tracking control for high-order nonlinear Cybernetics, vol. 46, no. 7, pp. 1591-1601, July 2016.

9. M. Fiacchini and I. Morrescu, "Convex conditions on decentralized control for graph topology preservation," IEEE Transactions on Automatic Control, vol. 59, no. 6, pp. 1640-1645, June 2014.

10. J. A. Fax and R. M. Murray, "Information flow and cooperative control of vehicle formations," IEEE Transactions on Automatic Control, vol. 49, no. 9, pp. 1465-1476, Sept 2004.

11. Z. Li, Z. Duan, G. Chen, and L. Huang, "Consensus of multiagent systems and synchronization of complex networks: A unified viewpoint," IEEE Transactions on Circuits and Systems I: Regular Papers, vol. 57, no. 1, pp.213-224, Jan 2010.

12. A. Zhang, S. Zhang, Q. Chen, and W. Zhu, "Consensus control of a class of second-order nonlinear multi-agent systems based on distributed adaptive protocol," in 2018 Chinese Control and Decision Conference (CCDC), June 2018, pp. 4668-4672.

13. X. Jin, S. Wang, J. Qin, W. X. Zheng, and Y. Kang, "Adaptive fault-tolerant consensus for a class of uncertain nonlinear second-order multi-agent systems with circuit implementation," IEEE Transactions semi-strict-feedback multiagent systems," IEEE Transactions on

on Circuits and Systems I: Regular Papers, vol. 65, no. 7, pp. 2243-2255, July 2018.

14. V. K. Yanumula, I. Kar, and S. Majhi, "Consensus of second-order multi-agents with actuator saturation and asynchronous time-delays," IET Control Theory Applications, vol. 11, no. 17, pp. 3201-3210, 2017

15. M. Guo and L. Liu, "Cooperative output regulation of a class of nonlinear uncertain multi-agent systems with unknown ecosystem," in 2015 IEEE International Conference on Information and Automation, Aug 2015, pp. 1252-1257.

16. H. Rezaee and F. Abdollahi, "Consensus problem over high-order multiagent systems with uncertain nonlinearities under deterministic and stochastic topologies," IEEE Transactions on Cybernetics, vol. 47, no. 8, pp. 2079-2088, Aug 2017.

17. X. You, C. Hua, and X. Guan, "Self-triggered leader-following consensus for high-order nonlinear multiagent systems via dynamic output feedback control," IEEE Transactions on Cybernetics, pp. 1-9, 2018.

18. C. W. Wu and L. Chua, "Application of graph theory to the synchronization in an array of coupled nonlinear oscillators," Circuits and Systems I: Fundamental Theory and Applications, IEEE Transactions on, vol. 42, no. 8, pp. 494-497, aug 1995.

19. M. Spong, "Review article: Modeling and control of elastic joint robots," Mathematical and Computer Modelling, vol. 12, no. 7, p. 912, 1989.

20. Z. Li, X. Liu, M. Fu, and L. Xie, "Global h1consensus of multi-agent systems with lipschitz non-linear dynamics," IET Control Theory Applications, vol. 6, no. 13, pp. 2041-2048, September 2012.

21. G. Wen, Z. Duan, G. Chen, and W. Yu, "Consensus tracking of multi-agent systems with lipschitz-type node dynamics and switching topologies," Circuits and Systems I: Regular Papers, IEEE Transactions on, vol. 61, no. 2, pp. 499-511, Feb 2014.

22. A. S. M. Isira, Z. Zuo, and Z. Ding, "Leader-follower consensus control of lipschitz nonlinear systems by output feedback," International Journal of Systems Science, vol. 47, no. 16, pp. 3772-3781, 2016.

23. Y. Hong, J. Hua, and L. Gao, "Tracking control for multi-agent consensus with an active leader and variable topology," Automatica, vol. 42, no. 7, pp. 1177-1182, 2006.

24. P. Pagilla and Y. Zhu, "Controller and observer design for lipschitz nonlinear systems," in American Control Conference, 2004. Proceedings of the 2004, vol. 3, 2004, pp. 2379-2384 vol.3.

\section{AUTHORS PROFILE}

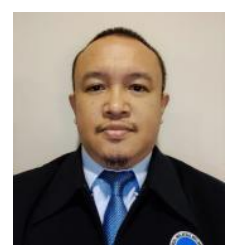

Ahmad Sadhiqin Mohd Isira received his Master of Engineering Degree in Electrical Engineering from University Teknology Malaysia (UTM), Malaysia, in 2008. He later obtained his $\mathrm{PhD}$ degree in Electrical and Electronics Engineering from Control System Center, University of Manchester, U.K in 2016. Currently he is a staff of Faculty of Electronics and Computer Engineering, University Teknikal Malaysia Melaka. His research interests include nonlinear control, complex network, cooperative control of multi agent systems, energy harvesting and automation control system.

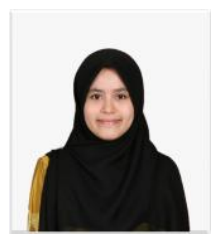

Nur Syahirah Hamdan received her Bachelor of Electronic Engineering with Honours from Universiti Teknikal Malaysia Melaka (UTeM), Malaysia, in 2019. She is currently working on her Master degree at UTeM. Her area of interests are consensus control, control system, optical communication and optical sensor.

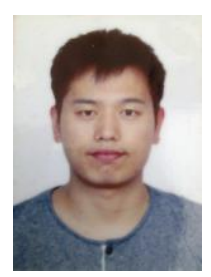

Chunyan Wang received the M.Sc. degree in electrical and electronic engineering from the University of Greenwich, London, U.K., in 2012, and the Ph.D. degree in control systems from the University of Manchester, Manchester, U.K., in 2016. He was a Research Associate with the School of Electrical and Electronic Engineering, the University of Manchester

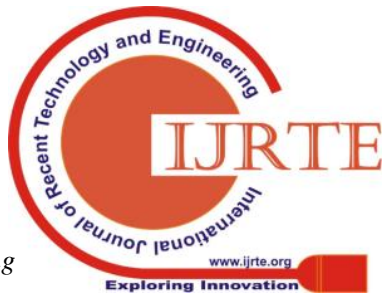


from 2016 to 2018. He is currently an Associate Professor with the School of Aerospace Engineering, Beijing Institute of Technology (BIT). His current research interests include cooperative control, UAVs, and robust control.

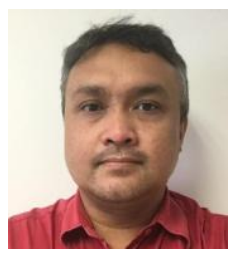

Mohd Hendra Hairi ( Phd, MIEEE, MIET) received his Bachelor's degree in Electrical Engineering from Universiti Sains Malaysia in 2001 and Master of Science degree in Electrical Power Engineering from Universiti Teknologi Malaysia in 2003. He received his Ph.D degree from University of Manchester in 2015. He has served as an academic staff at Universiti Teknikal Malaysia Melaka (UTeM) since 2003 and he is currently a senior lecturer in the Faculty of Electrical Engineering (FKE), UTeM. He was formerly the Head of the Energy and Power System group (EPS), FKE. He is an expert in power system protection and his research interests included power system, renewable energy and distributed generation. 